\title{
The Grand Unification of Dark Matters: The Dark Universe Revealed
}

\author{
Dan Sharpe \\ Fitzwilliam, New Hampshire, USA
}

Email address:

dbdotnet1@gmail.com

\section{To cite this article:}

Dan Sharpe. The Grand Unification of Dark Matters: The Dark Universe Revealed. International Journal of Applied Mathematics and Theoretical Physics. Vol. 3, No. 2, 2017, pp. 26-37. doi: 10.11648/j.ijamtp.20170302.12

Received: January 31, 2017; Accepted: February 15, 2017; Published: March 17, 2017

\begin{abstract}
A unifying model and theory that covers dark matter, dark energy, gravity, space and negative energy by means of observation, citation, and deduction, revealing a fundamental definition for both space and gravity, support of dark energy theory, explanation of dark matter, revealing additional structure and properties of baryonic atoms, sheds new light on baryogenesis, and revises estimates of the longevity of the universe. Other matters discussed are aerospace, anti-gravity, artificial gravity, gravitational waves, wormholes, interstellar space travel, cold fusion, cold fission, health and wellbeing, the earth's atmosphere and global warming.
\end{abstract}

Keywords: Space, Dark Matter, Gravity, Dark Energy, Hydrogen

\section{Introduction}

Dark Matter is the term used for the prevailing theory of the 1980's. Scientists needed a way of explaining how stars in galaxies rotated in a unified manner, unlike solar systems, where the closest planets orbited their star quicker than the outer planets. The other problem is most galaxies didn't appear to have enough mass to sustain the orbits of all their stars.

Some estimates go as high as $27 \%$ of the universe consists of Dark Matter.

Scientists have all but given up at finding Dark Matter Particles.

The Dark Universe consists of weak and strong interactions between space, gravity, matter and energy. Dark Matter has similar laws of physics, but space and gravity are weak to Dark Matter, something like what you would expect with a Shadow Universe. [1]

- Matter: Composite Atoms, comparable to the known periodic table and beyond.

- Particles: Largely indistinguishable with Cold Fission.

- Energy: Potential, Kinetic, Gravitational, Chemical, Fusion, Fission, Elastic, Motion, Thermal, Sound, Electromagnetic and Electric

- Interactions: Strong, Electromagnetic, Gravitational

- States: Gaseous, Liquid, Sold and Plasma
Amongst the most abundant elements in the universe are hydrogen, oxygen and carbon, all of which are the building blocks for water and life. This would suggest a wide variety of life is prevalent throughout the universe.

Ordinary hydrogen (proton) is by far the most common atom in the universe and makes up about $99.985 \%$ of all naturally occurring hydrogen atoms. It is the only stable element known to man that doesn't have a neutron in its nucleus. All it takes is a single proton, or a single proton and electron(s), to make a hydrogen atom, also known as a protium isotope. [2]

Protons have an electron under normal temperatures and pressures, which is very chemically reactive. Hydride is a proton with two electrons, making it negatively charged. A lone proton is positively charged and easily combines with other atoms to form molecules, so it is rarely seen isolated except in solar winds. [3]

Hydrogen primarily exists in a diatomic gaseous state known as molecular hydrogen, which has numerous therapeutic benefits.

Burning hydrogen in air acts as a zero-emission fuel that reacts with the oxygen to release energy and forms water vapor.

Other stable forms of hydrogen exist that include one neutron in the nucleus, called a deuterium isotope, known as heavy hydrogen and used for heavy water, and two neutrons in the nucleus, known as a tritium isotope which is 
radioactive, often a byproduct of nuclear reactions. This deuterium-tritium pair has great potential for next generation large-scale fusion power plants to supply the world's energy needs, all from ocean water. The ITER Tokamak complex is under construction and by 2027 is expected to produce more energy from the fusion plasma process than is used, which has not been achieved in any fusion reactor. NASA has similar plans for deuterium-tritium pair fusion plasma reactions to speed their first man mission to mars.

Hydrogen appears pale blue in a flame, but with space photography, it really depends on how the filters are applied to colorize the photo, so oxygen may appear blue, while hydrogen may appear green. Often with distant galaxies, a broadband spectrum is used, similar to what the naked eye would see, but with most photo's it really depends on which color palette is being used in the processing of the photo.

The universe consists largely of hydrogen, but modern telescopes are only able to observe exited hydrogen atoms, using a color spectrum filter, such as those atoms around an exploding star, in a gas cloud, or after receiving energy wavelengths or photons from a nearby source.

The Wilkinson Microwave Anisotropy Probe (WMAP) was monitoring the cosmic microwave background fluctuations of the early universe for several years, and has provided better statistics about the types of matter in the early universe. [4]

The European Space agency's Planck Probe has completed its census of the universe and its showing more matter and less energy, by as much as $4 \%$ of what was expected, which puts Dark Matter at $26.8 \%$, ordinary matter at $4.9 \%$, with a $4 \%$ drop in the amount of Dark Energy. [5]

\section{Dark Matter Primer}

The simplest explanation for Dark Matter is that it is mostly hydrogen, but how is that any different than what we already know, other than it hasn't been proven by science yet? According to the Los Alamos National Laboratory, hydrogen makes up more than 90 percent of all the atoms.

Dark Matter is very much like hydrogen, and certainly includes hydrogen, with a single proton and electron(s). However, more complex atoms are formed without neutrons as part of the big bang, exploding stars, and with Cold Fusion, in ways where the atom is not only transparent, has less mass, and has significantly less weight as a result of gravity.

It is generally regarded that for atoms to be stable, multiple positively charged protons need neutral neutrons in the nucleus to act as a buffer, even though they are bound with the strong force and balanced with negatively charged electrons in orbit.

All atoms overcome the repulsion of the positively charged protons and negatively charged electrons, with the strong force, similar to how mesons work to bind all stable atoms.

Science generally considers larger atoms without neutrons to be unstable, however, these atoms don't reflect light, are difficult to observe even under the best of circumstances, are lighter than ordinary matter, and who ever said Dark Matter was entirely stable anyway?

Protons must be extremely close for the strong force attraction to occur and overcome the repulsion of two or more positively charged protons. However, kinetic energy and pressure plays a much larger role with Dark Matter fusion than heat in stars, so Cold Fusion becomes possible, and Cold Fission is more readily achievable.

In terms of the big bang and exploding stars, atoms would eventually favor their simplest expression of ordinary matter, Dark Matter or splitting apart, mainly from collisions and proton-neutron transformations, so radiation from Dark Matter atoms, including hydrogen atoms, must be rare compared to ordinary matter atoms. Ordinary matter is largely detectable; however these Dark Matter atoms are mostly composites of two or more dark atoms that are eluding researchers.

It's completely understandable that science hasn't been able to detect Dark Matter particles, but they are obviously looking in the wrong place, because Dark Matter atoms and Dark Matter life are all around us. This may simply be a result of more stable molecules when binding these atoms or even more exotic states of matter that are yet to be discovered.

Dark Matter life is capable of passing through what we would otherwise consider ordinary matter with very little resistance, which is probably the best argument for even more exotic states of matter and perhaps a better and expanded understanding of quantum electrodynamics theory. [6]

Magnetism arises from the motion of the electrons in an orbit around the nucleus, similar to planets in a solar system, along with the rotation of the electron on its axis, similar to the rotation of a planet, making each electron like a tiny magnet.

Certain crystal like solids with the right molecular structure have been shown to be capable of passing through each other, but for common Dark Matter solids to pass through common ordinary matter solids, it would take more than just the orbits of the electrons to be in a ground state to avoid resistance and entanglement of the atom and molecular bonds in proximity.

Any molecular bonding between atoms involves a number of complicated interactions with different attractions and repulsions of a positive nucleus and negative electrons, with the space between the nucleus being negative, so the real question is do the attractions win and it all holds together or does the repulsion win and the molecule flies apart?

With Dark Matter, this becomes enormously more completed, to have two different and distinct forms of matter pass through each other without disruption of the other at a molecular level. This would be the fundamental basis to prove any Dark Matter theory, either by observation or mathematically, but it might actually help if we could see Dark Matter solids with our own eyes or with scientific instrumentation.

The answer that nature and the universe always resorts to 
is the simplest expression or representation of an object to hold and balance its form, where "simplest" means least expensive, therefore there would need to be a fundamental difference in polarity with electricity and electrodynamics of Dark Matter atoms and/or molecules for any Dark Matter theory to work, otherwise that theory will simply fly apart too.

In any case, Dark Matter is almost virtual and even holographic compared to ordinary matter, yet many of the physical laws still apply, with some phenomena, such as wormholes, occurring with great frequency. Since space is weaker to Dark Matter, interactions between Dark Matter and ordinary matter solids may not be as significant after all, it is simply a matter of space giving way. This does not necessarily cover every scenario though, such as a solid within a solid, so perhaps the simplest test of all would be to determine the polarity of baryonic atoms (ordinary matter) [7] without neutrons.

Exotic Matter has the same problem too, requiring a negative polarity, with a neutral, non-baryonic buffer in the nucleus of the atom to support life. That is by far the ultimate test of any Dark Matter theory. Aside from supporting life as we know it, some life in the Dark Universe is very similar to life in the Light Universe, so where did it start first, was it a duel evolution, or is there any conceivable way life could crossover during its development?

Perhaps some form of Exotic Matter does exit that works here, but it would have to be formed as part of the big bang, in a star or synthetically, and is not likely to have the right polarity or support Cold Fusion, which is a given and really needs a simpler expression of an atom to occur, so between gravity, space, polarity and Cold Fusion, the only possibility that remains is Dark Matter. Any number of forms of Exotic Matter could exist that result in bazar phenomena, but it's not likely to support life either. [8]

So does $E=\mathrm{mc}^{2}$ apply to Dark Matter? [9] The only difference is lower mass, so the potential energy is less, but Dark Matter is much more abundant to fuel the universe for far longer than the 5 billion years estimated by some scientists; it's more like 78 billion years of Dark Matter fuel, never mind exploding stars as a source of fuel, so upwards to 100 billion years before fuel runs out considering expansion and black holes, and upwards to another 100 billion years before the lights go out for all the smaller stars.

\subsection{Dark Energy and Gravity}

The other dark mystery that astronomer's came up with is Dark Energy.

In the 1990's, two scientific teams were measuring the distance and speed that galaxies were moving by studying super nova explosions. They were expecting to find that the speed of these galaxies was slowing since the big bang occurred, but they actually discovered they were accelerating. The prevailing theory is called Dark Energy; the stretching of empty space with an anti-gravity effect.

The notion of anti-gravity is currently in the realm of science fiction, but it stands to reason that mass results in gravity, so the lack of mass is neutral and the stretching of empty space could result in a negative gravitational effect, or does that imply the compression of space contributes to what we perceive as gravity?

Therefore, the simplest expression of gravity would be the atom actually "compresses space" enough to generate a minute gravitational field, so mass alone only indirectly results in gravity. Dark Matter has less mass and space is weak, or should I say space is compressed much less, and therefore has a lower gravitational weight. In short, the "displacement" of space by whatever means is gravity.

That makes the neutron the significant influence for gravity (displacement of space expected) and is responsible for light refraction and potential for radiation, while the proton-electron pair has some influence over gravity (positive and negative gravitational fields expected) and determines color and dampens radiation.

The Dark Energy theory has much support, such as the Cosmic Inflation theory [10] that resulted during the big bang. The other science fiction notion of doing space travel with an artificial gravitational field now becomes in the realm of possibility too, given enough neutrons or energy to displace space. That really opens the door to space too, getting large payloads into orbit. The most efficient way to do this would be further research into calibrating the displacement of space effectively.

With the Alcubierre Warp Drive theory, [11] by contracting space in front of you while expanding it behind you could in theory allow a spacecraft to travel 10x the speed of light, but notice the craft itself isn't actually moving, so no physical laws are broken, although such a drive is supposed to only be possible with the existence of Exotic Matter that has a negative Energy Density. That sounds a lot like Dark Matter, doesn't it? This theory is on the right track; a gravitational drive would however need to "Compress" space (strengthen gravity) "behind" it to ride the displacement wave, but there is potential of some drag from gravity. Placed in front of it would yield the attraction of gravity, but there is no way to avoid the space displacement waves. Expanding space behind it should work smother depending on thresholds that would need to be studied. In any case, that's fine for getting around the neighborhood, but a gravitational jump drive would be more practical, expanding space (weakening gravity) in front of the craft until a wormhole forms, adjusting power for the right distance, and then sliding right through.

The graviton is a theoretical particle that mediates the force of gravitation, but has yet to be discovered and has an outstanding mathematical problem with renormalization; therefore models beyond quantum field theory, such as string theory, have prevailed.

With this grand unification model, the electron bound to the neutron produces a significant gravitational influence (displacement of space), while the proton with free electrons, has a minor gravitational influence (positive and negative gravitational fields) that is expected to vary between ground and excited states, which has inconclusively been 
demonstrated with some hydrogen and gravitational red shift experiments, atomic structure, nuclear spin, partially dark atoms and even molecular structure. Perhaps it's easier to relate to the fabric of space in terms of being anti-gravity for the most part.

Albert Einstein determined that space-time was interwoven and the presence of mass "warps" space-time geometry, which results in gravity. [12-13]

The Gravity Probe-B confirmed two predictions of Einstein's theory of relativity, including frame-dragging, the spin of a large body distorts the space-time around it, and gravitational lensing, where light around a large body is bent. [14]

Many publications did a 100 year tribute to Albert Einstein for predicting gravitational waves that would one day be detected, and scientists recently detected them between two black holes, which is an amazing prediction for someone of that era. These photos clearly look like gravitational displacements on the positive side for black holes in proximity, but one photo in particular of two distant black holes looked more like a wormhole on the positive side, but that might have been an illustration and unfortunately sciencemag.com no longer has that photo on their site. In any case, it would take black holes to create a wormhole on the positive side. Gravity waves on the negative side would be much more subtle and an Einstein-Rosen bride on the negative side would take an extremely small fraction of that energy to produce, relative to the distance. [15-16]

The "Universal Polarities" (new nomenclature) are expected to be:

- Neutrons: Antineutrons - Protons (Hydrogen ortho nuclear spin): Antiprotons - Antiparticles - Hot Electrodynamics - Hot Thermodynamics (Positive)

- Protons (Hydrogen para nuclear spin) - Dark Protons Dark Neutrons - Cold Electrodynamics - Cold Thermodynamics (Negative)

Although warping, bending and displacing space-time are all basically the same concept as it applies to time, gravity is another story, especially since its mechanics are yet to be proven physically. The real question is; 1) does mass generate a gravitational field, 2) does mass warp space-time geometry to reduce the density of an anti-gravitational field on the planet, or 3 ) is there some combination of both with relative gravitational field densities that are yet to be determined?

Although \#1 is the general perception of gravity and is in line with Newton's observations, \#3 is expected in this model, because it's consistent with Dark Matter, and the electron supports two opposing fields with both a minimum and significant influence over gravity, plus for the sake of having an open theory and model that goes between Newton and Einstein that supports one, the other or both, however \#2 is the bias for the simplest expression of gravity, yet it is also the most difficult to comprehend, and is more in line with Einstein's predictions of a warp of space-time geometry.

The distinction that isn't immediately apparent is that the displacement of space is influenced by both mass and the fabric of space itself. [17]

The fabric of space is largely Dark Matter composite atoms and Dark Matter molecules, consisting of predominately negative gravity; however the density of this gravitational field is yet to be determined and these atoms have been somewhat allusive. [18]

In any case, Dark Matter has positive and negative forms of atoms and is expected to have positive and negative forms of gravitational fields with a negative prevalence, which is in line with \#3 and is not mutually exclusive of Einstein's predictions, [19] however in the event the atom (electron) only has one polarity related to gravity, it's conceivable that Dark Matter is different than ordinary matter by having two polarities, while ordinary matter only has the one polarity, excluding antimatter, so this could go either way between Newton or Einstein, and there is always the possibility they are both right for the most part, since atoms have both neutrons and protons.

In simplest terms, mass is positive to gravity and space is negative to gravity overall, but both geometrical systems overlap to the core of a planet or star, because mass contains composite atoms of neutrons and protons, and protons represent both mass and space under this model, supporting the conclusion that the neutron (bound electron) displaces space and the proton is positive gravity where mass is involved and negative gravity in space for the most part, while Dark Matter, partially Dark Photons and variations in the electron orbit are negative gravity.

In any case, the mechanism for influence over gravity is expected to be the electron as described above and what is really needed is solid research to conclusively demonstrate the dynamics of gravity, which is the key to recognizing which research, theories and models of the universe are on track, and will truly open the door to space and interstellar exploration.

Other potential influences on gravity include the spin of the planet resulting in lower gravitational weight at the equator [20], the electromagnetic field of the planet or star [21], the tide bulge of our moon [22] and the momentum [2325] and electromagnetic [26-27] stress-energy tensors.

Historically, the space time perspective was a twodimensional plane, with three-dimensional space, with time as the fourth dimension. [28] Minowski simplified this perspective, and subsequently a large number of physical theories, by making time and space inseparable, as a unified dimension. [29] Although this perspective makes the math easier, along with other "good enough" shortcuts like Newtonian generalizations, this theory strives to define and explain the model, its foundation, its framework, its structure, its properties, its dynamics, and its mechanics, with enough references to let everyone have at it.

This model views ordinary matter (neutron-proton atoms) as the basis for three-dimensional space, whereas space-time is the fourth dimension (proton-dark atoms). All of this is a matter of perspective to illustrate how space is weak to Dark Matter (wormholes) and to make some distinctions about ordinary matter in the coming sections, all of which does not 
involve time [30], none of which are easy concepts to express with modern physics, since Dark Matter is the fabric of space.

The nomenclature for a wormhole implies a tunnel and has science fiction connotations, whereas the Einstein-Rosen bride or a hole in space is more accurate, which occurs with great frequency with Dark Matter, without involving time in regards to Dark Matter, which is not supposed to be the case with ordinary matter and space-time. Space-time nomenclature needs to change because on the positive side with ordinary matter activities, like rocket propelled ships, space-time doesn't change, but it becomes infinity on the negative side, so interstellar travel is just a quick jump away on the negative side, which doesn't involve time either. [31]

The "Gravitational Polarity" (new nomenclature) is expected to be the same as the Universal Polarity, with the significant influence being mass as a displacement of space (positive polarity) and space being a negative polarity, which is in line with Einstein's projection of warped space-time geometry and Newton's observations, however it represents two overlapping gravitational forces and geometric systems.

Positive:

- Significant Influence: Neutrons as a displacement of the fabric of space.

- Minor Influence: Protons as a positive gravitational field.

Negative:

- Minor Influence: Protons and Dark Protons as a negative gravitational field.

- Minor Influence: Dark Neutrons as a positive gravitational field.

Overlapping gravitational field densities are yet to be determined. The photon and dark matter field strengths fluctuate based on nuclear spin rotations, atomic structure, electron ground to excited states, and the molecular binding of molecules. Dark matter is expected to have a negative gravitational field for Dark Protons and a positive gravitational field for Dark Neutrons, which is expected to have similar variations in field densities.

There are a large number of Dark Energy theories that are not necessarily mutually exclusive. [31-35] The fabric of space, consisting of Dark Matter Molecules, in gaseous, liquid, sold and perhaps even dark plasma states, [2] is sufficient to explain both Dark Energy and Dark Matter related astronomy, astrophysics and cosmology related observations, but is not mutually exclusive either. [36-42] Although the following section only describes the two most common forms of Dark Matter atoms, it doesn't preclude others, including the pion, SIMP, WIMP, axion, MACHO, Kaluza-Klein, gravitino, and any other supersymmetric particles. [43]

Some estimates go as high as $73 \%$ of the universe consists of Dark Energy.

\subsection{Dark Matter}

Proton atoms are considered both ordinary matter and Dark Matter, but in actuality only certain proton atoms are partially dark, based on nuclear spin and photons that are fused to dark atoms. Protons support even extreme molecular structure [44-47] and the known states of matter [2], but have the benefit of being observable, permitting experimentation and having thousands of supporting references that also apply to Dark Matter. Hydrogen demonstrates the fabric of space without getting in to composite Dark Matter atoms, which obviously has greater molecular structure and predominately negative influence.

A proton atom with a negative polarity potential would have similar poles reversed, such as negative energy and negative thermodynamics, without a full inversion that results in an antiproton. These atoms would get smaller and colder as they bond (Dark Matter).

Aerospace fuel can largely be collected in space with a better understanding of the Cold Fission process.

We often think of space in terms of infinity, so we may need to start thinking of the longevity of the universe in those terms too. Depending on the thresholds of space, the Dark Matter periodic table has the potential to go to infinity with these atoms, indicating the periodic table for ordinary matter will increase substantially with further research.

Hot fusion in a star causes two proton atoms to bond, flipping one of them to a neutron, resulting in a release of hot positive energy, while the big bang and cold fusion results when two or more protons bond, achieving a new and smaller dark composite atom, with the release of cold negative energy.

With lighter atomic weights, fusion puts out more energy than it takes, but with heavier atomic weights, it takes more energy to result in fusion.

These hot fusion reactions also produce antimatter and antiparticles, including antiprotons, antineutrons, and positrons, which have an inverse spin and charge, so when they come in contact with ordinary matter and particle pairs, they annihilate each other resulting in a release of hot energy in the form of photons.

Since ordinary matter has both a positive and negative counterpart, with a positive prevalence, it stands to reason that Dark Matter would be the same; however, it would be a negative prevalence, which may shed light on baryogenesis. [48]

The structure of Dark Matter atoms would consist of multiple electrons orbiting the atom, similar to how all baryonic atoms work, where the spin isomer of the nuclei is reversed, which is demonstrated with molecular hydrogen (para and ortho) [49-50] at lower temperatures, and begins to explain the difference in dark matter electrodynamics [51], and the process for which the composite atom actually gets smaller; [52-53] such as 1 quark flipping, resulting in 3 up quarks, however the estimated delta lifetime [54-56] of such an atom in isolation is estimated at under a second, with no reference of a lifetime for the atom bonded with a proton. A composite dark atom is expected, where both protons eventually flip. A similar hot fusion reaction occurs with Helium-2 (diproton), which is a proton-proton chain reaction, with kinetic energy being the most important factor for both 
hot and cold reactions. [57-59]

In isolation, even the neutron only lasts 15 minutes, but fusion with a proton extends that free decay time. [60]

Conversely, positive Dark Matter atoms would have 3 down quarks, making the atoms significantly larger. Therefore, negative atoms (Dark Proton variants) would pass through ordinary matter with little to no resistance; however molecular structure could cause resistance, while positive atoms (Dark Neutron variants) could interact with ordinary matter and molecules. These interactions are weak and strong depending on the atoms and molecules involved. You might be surprised to learn your own body has Dark Matter components that are as much a mystery to modern medical science as Gravity, Dark Energy and Dark Matter is to physics. [61]

Aside from protons, it's not clear if any atoms in the Dark Matter periodic table have ever been observed by any scientific means, for any length of time, and yet they not only do exist, there lifetime is expected to be similar to protons, which is between $10^{\wedge} 29$ years to completely stable (Infinity), but Dark Matter will decay to protons and positive pions (Cold Fission) under the right environmental conditions. [6263]

Perhaps the simplest validation of this model and theory can be achieved using empty containers under different environmental conditions, to measure the amount of protons, pions, muons, photons, neutrinos and ions, which could vary depending on the decay method, that appear as a result of Dark Matter Cold Fission over time, taking ordinary matter fission into account.

In every conceivable way, the proton has potential for both positive and negative reactions and energies; however, it's important to recognize that negative energy is not photons (possibly a dark photon), goes well beyond negative ions, has its own negative energy density, includes negative gravitation, and could be described as cold thermodynamics. [64-70]

It's important to recognize that hydrogen will continuously escape to space at a rate of $3 \mathrm{~kg}$ per second on earth, so increased levels of hydrogen in the atmosphere are achievable with a reduced planetary mass and increased and continuous Cold Fission. Likewise, larger planets have hydrogen trapped in their upper atmospheres, with environmental conditions being equal, won't have as much Cold Fission occurring relative to their size, because more Dark Matter is displaced, but these planets also have a stronger gravitational attraction of hydrogen. Jupiter's atmosphere is $75 \%$ hydrogen, largely consisting of molecular hydrogen. [71]

Therefore, the neutron-proton atoms and the proton-dark atoms represent two distinct dimensions, and their union represents a balance of polar opposites between hot and cold, light and dark, ordinary matter and Dark Matter, positive energy and negative energy, hot fusion and Cold Fusion, hot fission and cold fission, radiation and anti-radiation, gravity and anti-gravity (Dark Energy), and space, with the latter being the proton-dark atoms and anti-space being the neutron-proton atom. Both of these dimensions require further exploration and sold research to reveal all of their mysteries, and how interactions with other atoms (dimensions) occur.

Each form of matter discovered has potential to be a dimension of its own, perhaps with similar or entirely different laws of physics, with life or phenomena, and their exploration may reveal additional interactions with the known universe that could even break the laws of physics as we now know them.

The prevailing theories in quantum physics are particlewave oriented:

- The Standard Model, with over 40 species of elementary particles that can combine to form composite particles, but doesn't preclude the existence of multiple dimensions.

- String Theory, with essentially one type of particle, having multiple residency frequencies, with 11 different dimensions.

Each form of particle, including gamma rays, cosmic rays, photons, neutrinos, quarks, muons, etc., and composite particles such as neutrons, protons and electrons, could result in additional interactions with the known universe, with other dimensions, with other atoms and particles, and may help predict the evolution of the universe as she matures.

As you look further across the visible universe, you look further back in time, all the way back to the big bang, which occurred about 13.8 billion years ago. Since you can't look beyond the beginning of time, the vast majority of the universe cannot even be seen, making the Milky Way galaxy a mere speck in the scheme of things.

Depending on whom you listen to, this known universe only accounts for $5 \%$ to $25 \%$ of the entire universe, making our galaxy, with 100-400 billion stars and a 100,000-180,000 light year diameter, much less than a speck within a speck, and our solar system much less than a spec within that.

About 95 to $96 \%$ of the known universe consists of Dark Matter and Dark Energy, so the Light Universe that we think of as reality is a mere 4 to $5 \%$ of the known universe, which makes her very young, more like an infant.

\subsection{Dark Matter Health Impact}

Even though instrumentation to detect this Dark Universe doesn't exist yet, there is a way to observe it...

Humans have evolved with this Dark Universe. With some practice and experience it can be observed through the mind's eye; mostly in the form of color, shape and density. In some circles, this form of energy is referred to as Cosmic Energy, not to be confused with cosmic rays from exploding stars, which is the vapor (gaseous) form of Dark Matter. The actual translation of Shakti means "Empowerment" or "Power" as dynamic forces that move through the entire universe. [72]

Cosmic Energy is accompanied by the cold release of negative energy in the body as Dark Matter breaks down (Cold Fission), so hydrogen, negative energy and negative ions are essential for life, health and wellbeing. The term 
Cosmic Energy goes back about 3,700 years in the written record, but who knows how long mankind has recognized this primal energy of the universe as being essential for vitality.

The body is capable of running hot for extended periods of time, but it comes at a price. Striving for balance, with the ability to run hot (positive energies) and/or cold (negative energies) as needed is ideal, which is often a challenge with all the stressors of modern society, at times of illness, and with the elderly in particular, considering current atmospheric and environmental conditions.

Drinking water and eating carbohydrates gets you part of the way there, although hydrogen absorption is limited to metabolic reactions in the body yielding a neutral charge, whereas pure hydrogen in the atmosphere and Dark Matter from the environment results in a cold reaction in the body that yields even greater negative energy as it breaks down.

Several hundred scientific articles demonstrate diatomic (molecular) hydrogen to have therapeutic potential in every organ of the body and against hundreds of diseases, including the reduction of oxidative stress and oxygen radicals, upregulation of antioxidant enzymes, anti-inflammatory effects, anti-apoptotic benefits, anti-obesity effects, and antiallergy benefits.

Therefore, anyone who needs oxygen in particular should have it accompanied with molecular hydrogen gas, or better yet, use of Dark Matter gas in place of hydrogen. [73-74] This is by far the best delivery method of molecular hydrogen.

The average hydrogen content in the air at ground level is. 6 parts per million, which varies and the testing methods sound like they need review, but more recent studies have concerns about molecular hydrogen being highly reactive at increased levels and could cause interactions with pollutants in our atmosphere, so the real question is how could molecular hydrogen be doing any real harm in the atmosphere, when there is significant medical research demonstrating it to be very beneficial in the body? [75]

This needs further study to ensure reduced molecular hydrogen levels in the atmosphere wouldn't actually speed global warming, along with further evaluation of the escape rate of molecular hydrogen to space, and determine what pollutants would have an easier or harder time flushing out of the body, or for that matter, falling back to the ground and ocean level environments, how long it would take for them to break down one way or the other over time, and are there really any significant issues here, aside from having pollution itself? This is way beyond of the scope of this paper and aside from analysis and summation; it may even lend itself to modeling of the data over time.

The irony here is the most abundant atom in the universe is in scarce supply when it comes to fully supporting overall health and longevity on planet earth. It's almost like we were built for a smaller planet.

Other things that have a partial benefit include connecting a patient to a negative energy power source (doesn't exist yet), or use of a molecular hydrogen water generator that ionizes drinking water (marginal benefits), taking a bath in water rich in molecular hydrogen, increasing the production of certain intestinal bacteria, or use of a negative ionizer device in the room, similar to the Wein VI-2500 design (450 trillion ions/sec), because many of these devices on the market today have questionable functionality and concentrations. [76]

Negative ions have several hundred scientific articles to support their benefit. They are often found naturally near running water, like a stream and after a storm, or while taking a shower. [77-79]

Hydrogen, Dark Matter, Negative Energy and Negative Ions will certainly be areas of further study.

\subsection{Global Warming}

Global Warming is a complex matter that we definitely want to get right the first time and it probably still needs much study, but in the shorter term, what we should evaluate is a partial stopgap solution. Here is an over simplification of the process, but keep in mind that with hot fusion reactors, that put out more energy than they consume, are over 10 years away and that's after decades of evaluation.

Construct facilities that would use the ocean as a source for water to separate the hydrogen-oxygen atoms by the most efficient means, without any secondary reactions from the salt. Release the oxygen in to the atmosphere and continuously compress the hydrogen atoms (Cold Fusion) in tanks, harnessing negative energy from the tanks which hopefully can be made as simple as drawing negative current; the Dark Matter should get released through the tank (outside tanks) and get put back into the environment. In the event the tank starts floating, determine the next lowest density alloy that doesn't have secondary reactions to hydrogen.

As these Dark Matter atoms naturally breakdown, they will eventually help to reduce global temperature at the poles, improve hydrogen levels in our atmosphere, and put negative energy back into the environment, but the immediate benefit is to start reducing sea levels provided most countries participate. Much study is needed with Cold Fusion, Cold Fission, Dark Matter and transforming negative energy, but with enough efficiency, this could be a fairly clean and continuous operation, with various avenues for profitability.

The planet has a fixed surface gravity of $9.81 \mathrm{~m} / \mathrm{s},[80]$ so ultimately anything we do with Dark Matter on any kind of scale could cause a displacement that will end up in space, it's more a matter of when; however the mass of the planet is also being reduced which allows for more space (Dark Matter) distributed across the planet.

Further study is needed to determine atmospheric factors, displacement factors, temperature factors, weather impact, and best geographical locations for these facilities to get the most benefit and ensure the naturally occurring fission reactions occur planet side in a timely manner (use of the right alloy), which may mean building smaller facilities across the planet rather than larger ones in fewer sites.

It's mostly a matter of capacity, energy output, forecasting, timing and impact, which should be modeled for optimum 
performance.

Hydrogen is highly reactive, so the molecular binding of water molecules needs further study with increased oxygen and hydrogen levels in the atmosphere to effectively determine any net change in fresh water rainfall and snowfall and planetary mass over the long term.

Any process involving hydrogen and its compression is a challenge, both in terms of process and cost. Although Cold Fusion occurs to some degree with any lower temperature tank compression, achieving large scale production involves taking into account factors like kinetic energy, pressure, temperature, density, viscosity, and molecular binding, including solids, liquids and gaseous states of hydrogen, along with ionization and perhaps other factors, [81-91] that may take years to conclusively demonstrate Cold Fusion Dark Matter production to scale and effective Cold Fusion hydrogen production to scale and potentially inexpensive Cold Fusion hydrogen production to scale.

\section{Results and Discussion}

We live in a parallel universe with at least two known dimensions, the proton-dark dimension $\left(4^{\text {th }}\right.$ dimension $)$ and the neutron-proton dimension $\left(1^{\text {st }}-3^{\text {rd }}\right.$ dimensions $)$ that are polar opposites. Each dimension has its own "similar" periodic table, predominate energy polarity, predominate gravitational polarity, energy density, fusion, fission, and supports life; ordinary matter life and dark matter life. These dimensions are co-dependent, where if either were significantly depleted or decayed, it would be another big bang.

The longevity of the universe is now 200 billion years at a minimum, but will likely be $100 \mathrm{x}$ that to infinity, with an ongoing shift from a proton-dark based universe to a neutronproton paired universe.

- The neutron atom has the significant influence over gravity (displacement of space expected) and is responsible for light refraction and has the potential for radiation at accelerated time (free decay).

- The proton-electron pair atom has some influence over gravity (positive and negative gravitational fields expected) and determines color and dampens radiation at normal space-time (Infinity expected).

Although photons and Dark Matter (Dark Photons and Dark Neutrons) were largely produced during the big bang, despite multiple decay methods, and the presence of muons, there is no weak interaction, and no resulting radioactivity expected.

Hot fusion in a star causes two proton atoms to bond, flipping one of them to a neutron, resulting in a release of hot positive energy, while cold fusion results when two proton atoms bond, achieving a new and smaller atom, with the release of cold negative energy.

In every conceivable way, the proton has potential for both positive and negative reactions and energies; however, it's important to recognize that negative energy is not photons (possibly a dark photon), goes well beyond negative ions, has its own negative energy density, includes negative gravitation, and could be described with cold thermodynamics. [64-70]

The fabric of space consists largely of Dark Matter composite atoms and Dark Matter molecules.

Space largely consists of both Dark Matter and Dark Energy theory, along with hydrogen and other gasses, matter, energy and time.

Cold Fusion can be achieved by compressing hydrogen to Dark Matter, yielding negative energy; provided it's done properly with the energy drawn from the tank.

Cold Fission could be used to improve hydrogen levels in the atmosphere and reduce ocean levels and global temperatures, with a continuous flow of hydrogen rising to space.

Aerospace fuel can largely be collected in space with a better understanding of the Cold Fission process.

Gravity is the displacement of space by whatever means.

Artificial gravity in space can be achieved with sufficient neutrons, Dark Neutrons or energy to displace space.

Lifting payloads into space can be achieved with Dark Matter, provided Dark Matter can be contained in volume. Artificial anti-gravity on earth can be achieved with Dark Matter (Dark Photons) with the same provision.

Hydrogen, Dark Matter, Negative Energy and Negative Ions are essential to life, health, wellbeing, vitality, and longevity.

\section{Conclusions}

The fundamentals of the grand unification of dark matters model and theory is almost a certainty at this point, although some aspects of the model are somewhat speculative based on deduction and citation of unproven theories. It will require validation to spark significant research and advancements in the areas of physics, astrophysics, cosmology, energy, health, climate and various applied sciences.

This model offers a potential for a clean stopgap solution to global warming that has a beneficial effect on water levels, oxygen levels and hydrogen levels in oceans and in our atmosphere over time.

Humans have the potential to live to 150 years old, but we obviously don't have enough hydrogen in our atmosphere to support our overall health and longevity. This needs further study, but the focus should start with hospitals and with the elderly, to get the right mix of oxygen and hydrogen. According to the Royal Society of Chemistry, hydrogen is essential for life and is present in nearly all the molecules in living things. According to Dr. Patrick Flanagan, hydrogen may be the missing link to slowing down the aging process.

As it turns out, there is more to this than just hydrogen in the atmosphere and in our drinking water that is covered throughout.

\section{Nomenclature: (wikipedia.com)}

Air Ionizer (1999-2016), Alcubierre Warp Drive Theory 
(1994), Anti-gravity (1889-2014), Antihydrogen (19832016), Antineutron (1956-2019: Two down antiquarks, one up antiquark, with bounded positron), Antiparticle (19302011), Antiproton (1928-2015: Two up antiquarks, one down antiquark), Atmospheric Escape (1961-2012), Baryogenesis (1928-1999), Baryon - Baryonic Matter (Ordinary Matter), Cold Fission (1989-2002), Cold Fusion (1989-2010), Cosmic Energy (1700 BCE-2016), Cosmic Inflation Theory (1980-2016), Dark Energy Theory (19902016), Dark Matter Theory (1922-2016), Delta Baryon (2008-2013), Diatomic Molecular Hydrogen (1961-2010), Diproton - Helium-2 (1968-2019), Down Quark (19642013), Einstein's General Relativity (1915-2016), Electromagnetic Stress-Energy Tensor (1973-2012), Electromagnetism (1873-2006), Electron (1838-2016), Energy Density (Ordinary Energy), Exotic Matter (19682016), Free Neutron Decay (2004-2012: 15 Minutes), Graviton (1973-2014), Gravity (1638-2016), Hydrogen (1766-2011), Hydrogen Atom (1919-2011), Hydrogen Fuel (2005-2015), Hydrogen Ion (1997), Ionization (1966-2013), Ionization Energy (1988), Mesons (1934-2008: quarkantiquark pair), Metallic Hydrogen (1935-2017), Milki Way (1715-2015), Negative Energy (1997-2014), Negative Ions (1834-2013), Neutron (1920-2016: Two down quarks, one up quark, with bound electron), Newton's Law of Universal Gravitation (1729-2009), Nuclear Fusion (1950-2014), Pion - pi meson (1950-2018), Positron (1928-2016), Proton (1920-2016: Two up quarks, one down quark), Protonproton chain reaction (1939-2012), Quantum Electrodynamics Theory (1920-2016), Quantum Mechanics (1900-2016), Quark (1961-2016), Quark Model (19642004), Renormalization (1944-1990), Space-Time (19081973), Spacetime (300 BCE-2014), Spin Isomers of Hydrogen (1986-2015), Standard Model (1970-2016), Stress-Energy Tensor (1973-2005), String Theory (19602016), Strong Force (1983-2012), Surface Gravity (19702015: $9.8 \mathrm{~m} / \mathrm{s})$, Thermodynamics (1849-2010), Up Quark (1961-2012), Wormhole (1935-2012)

\section{References}

[1] Powell, C (2013) The Possible Parallel Universe of Dark Matter, Discovery Magazine [Online]. Available: $\mathrm{http}: / /$ discovermagazine.com/2013/julyaug/21-the-possibleparallel-universe-of-dark-matter.

[2] Jordon (2007) Hydrogen Fundamentals, HySafe [Online]. Available:

http://www.hysafe.org/download/997/brhs_ch1_fundamentalsversion\%201_0_1.pdf.

[3] (2017) Dummies Guide to Hydrogen, Molecular Hydrogen Foundation [Online] Available: http://www.molecularhydrogenfoundation.org/coreinformation/dummies-guide-to-hydrogen-gas/.

[4] (2014) What is the Universe Made of? NASA VAMP Wilkinson Microwave Anisotropy Probe [Online] Available: https://map.gsfc.nasa.gov/universe/uni_matter.html.
[5] Tuttle, K. (2015) Planck Satellite Brings Early Universe into Focus (Kavli Hangout), Kavli Foundation [Online] Available: $\mathrm{http}: / / w w w . s p a c e . c o m / 28570$-planck-satellite-brings-earlyuniverse-into-focus.html.

[6] Choi, C (2016) Dark Matter's Invisible Hand, Nova Next [Online] Available:

http://www.pbs.org/wgbh/nova/next/physics/interacting-darkmatter/.

[7] W.-M. Yao et al. (Particle Data Group) (2006). "Review of Particle Physics". Journal of Physics G. 33: 1-1232. arXiv:astro-ph/0601168. Bibcode:2006JPhG...33...1Y. doi:10.1088/0954-3899/33/1/001.7.

[8] Darling, D Exotic matter". Encyclopedia of Science, [Online] Available:

http://www.daviddarling.info/encyclopedia/E/exotic_matter.ht $\mathrm{ml}$.

[9] Einstein, Albert (1905), Mass-Energy Equivalence (1905). "Ist die Trägheit eines Körpers von seinem Energieinhalt abhängig?", Annalen der Physik, 18: 639-643, Bibcode:1905AnP...323..639E, doi:10.1002/andp.19053231314. See also the [Online] Available English Ttranslation:

http://www.fourmilab.ch/etexts/einstein/E_mc2/www/.

[10] Tsujikawa, Shinji (2003). "Introductory review of cosmic inflation": arXiv:hep-ph/0304257. Bibcode:2003hep.ph....4257T.

[11] Alcubierre, Miguel (1994). "The warp drive: hyper-fast travel within general relativity". Classical and Quantum Gravity. 11 (5): L73-L77. arXiv:gr-qc/0009013.

Bibcode:1994CQGra..11L..73A. doi:10.1088/02649381/11/5/001.

[12] Einstein, Albert (1915) Albert Einstein's Theory of General Relativity,"Die Feldgleichungen der Gravitation" Sitzungsberichte der Preussischen Akademie der Wissenschaften zu Berlin: 844-847 847 [Online] Available English Translation:

http://einsteinpapers.press.princeton.edu/.

[13] Read, N (2015) Einstein's Theory of General Relativity, space.com [Online]. Available: http://www.space.com/17661theory-general-relativity.html.

[14] Perrotto, $T$ (2011) "NASA's Gravity Probe B Confirms Two Einstein Space-Time Theories". Nasa.gov. Milgrom, M. 1983, ApJ, 270, 365 Bipcode: 1983ApJ...270..365M doi: $10.1086 / 161130$.

[15] The Discovery of Gravitational Waves, Scientific American [Online] Available: https://www.scientificamerican.com/report/the-discovery-ofgravitational-waves/.

[16] In Milestone, Scientists Detect Gravitational Waves As Black Holes Collide, the two-wave [Online] Available: http://www.npr.org/sections/thetwoway/2016/02/11/466286219/in-milestone-scientists-detectwaves-in-space-time-as-black-holes-collide.

[17] Nemiroff, R.; Bonnell, J., eds. (2014). "The Potsdam Gravity Potato". Astronomy Picture of the Day. NASA.

[18] M. Yu. Khlopov (2015) 10 years of composite dark atoms arXiv:1512.01081. 
[19] Xiang-Song Chen (2015) Co-existence of Gravity and Antigravity: The Unification of Dark Matter and Dark Energy arXiv:astro-ph/0506070.

[20] (2015) Gravity Variations, DataGenetics [Online] Available: http://datagenetics.com/blog/september62015/index.html.

[21] Ayre, J (2012) Magnetic Field And Gravity Intimately Linked, planetsave.com [Online] Available: http://planetsave.com/2012/10/26/the-magnetic-field-andgravity-from-a-satellite-perspective/.

[22] Strobel, N (2001) Gravity Interactions, Astronomy Notes [Online] Available:

http://www.astronomynotes.com/gravappl/s10.htm.

[23] Kobertein, $B$ (2014) Why Einstein will never be wrong, Universe Today [Online] Available: https://phys.org/news/2014-01-einstein-wrong.html.

[24] (2006) Huggett, Nick and Hoefer, Carl, (2006) Absolute and Relational Theories of Space and Motion, The Stanford Encyclopedia of Philosophy, Edward N. Zalta (ed.), https://plato.stanford.edu/archives/win2016/entries/spacetimetheories.

[25] Baez and Bunn (2006) The Meaning of Einstein's Equation, National Science Foundation [Online] Available: http://math.ucr.edu/home/baez/einstein/einstein.pdf.

[26] Gidfea, E. (2012) Electromagnetics General Theory of the Electromagnetic Field Classical and Relativistic Approached, Third Edition, Transylvania University Press [Online] Available: $\quad$ http://webbut.unitbv.ro/Carti\%20online/Fizica/Nicolaide.pdf.

[27] Fitzpatric, $R$ (2006) Relativity and Electromagnetism, University of Texis [Online] Available: http://farside.ph.utexas.edu/teaching/em/lectures/node106.htm 1 .

[28] Stover, Christopher and Weisstein, Eric W. Euclidean Space." From MathWorld--A Wolfram Web Resource. [Online] Available: http://mathworld.wolfram.com/EuclideanSpace.html.

[29] Petkov, V. (2010). Minkowski Spacetime: A Hundred Years Later. Dordrecht, Netherlands: Springer. p. 70. ISBN 9048134749

[30] Sanders, $R$ (2010) Most Precise Test Yet of Einstein's Gravitational Redshift, UC Berkly, [Online] Available: http://news.berkeley.edu/2010/02/17/gravitational_redshift/.

[31] Hogan, Jenny (2007). "Unseen Universe: Welcome to the dark side". $\quad$ Nature. 448 (7151): 240-245. Bibcode:2007Natur.448..240H. doi:10.1038/448240a. PMID 17637630 .

[32] Caldwell, R. R. (2002). "A phantom menace? Cosmological consequences of a dark energy component with super-negative equation of state". Physics Letters B. 545 (1-2): 23-29. arXiv:astro-ph/9908168. Bibcode:2002PhLB..545...23C. doi:10.1016/S0370-2693(02)02589-3.

[33] Kazuharu Bamba, Salvatore Capozziello, Shin'ichi Nojiri, Sergei D. Odintsov (2012) Dark energy cosmology: the equivalent description via different theoretical models and cosmography tests arXix: arXiv:1205.3421v3 doj: 10.1007/s10509-012-1181-8.
[34] Moffat, J. W. (2005), JCAP, 2005, 003 Gravitational theory, galaxy rotation curves and cosmology without dark matter Bipcode: 2005JCAP...05..003M doi: 10.1088/1475$7516 / 2005 / 05 / 003$

[35] Amendola, L and Tsujikawa, S (2010) Dark Energy $1^{\text {st }}$ Edition, ISBN: 0521516005.

[36] Xiang-Song Chen (2015) Co-existence of Gravity and Antigravity: The Unification of Dark Matter and Dark Energy, arXiv: astro-ph/0506070 Choi, c (2013) New Kind of Dark Matter that Could Form Dark Atoms, Space.com [Online] Available: http://www.space.com/21508-dark-matter-atomsdisks.html.

[37] Cartlidge, E. (2009) Is Dark Matter mostly 'Dark Atoms'?, PhysicsWorld.com [Online] Available: http://physicsworld.com/cws/article/news/2009/sep/21/isdark-matter-mostly-dark-atoms.

[38] Copi, C. J.; Schramm, D. N.; Turner, M. S. (1995). "Big-Bang Nucleosynthesis and the Baryon Density of the Universe". Science. 267 (5195): 192-199.

[39] Salucci P., Lapi A., Tonini C., Gentile G., Yegorova I., Klein U.: The universal rotation curve of spiral galaxies. II: The dark matter distribution out to the virial radius. MNRAS (2001) 323 (2): 285292 arXiv:astro-ph/0703115 doi j.1365-8711.2001.04077.x.

[40] Sobouti, Y., Hasani Zonoozi, A., Haghi, H.: Tully-Fisher relation, key to dark matter companion of baryonic matter. Astron. Astrophys, A\&A 507, 635-638 (2009) doi: 10.1051/0004-6361/200912571

[41] Garrett, Katherine; Dūda, Gintaras (2011). "Dark Matter: A Primer". Advances in Astronomy. 2011: 1-22. arXiv:1006.2483. Bibcode:2011AdAst2011E...8G. doi:10.1155/2011/968283.

[42] Kipreos (2005) Dark Energy Explained by Relativistic Time Dilation?, Astronomy Now, [Online] Available: https://astronomynow.com/2015/01/05/dark-energy-explainedby-relativistic-time-dilation/.

[43] Bergstrom, L. (2000). "Non-baryonic dark matter: Observational evidence and detection methods". Reports on Progress in Physics. 63 (5): 793-841. arXiv:hep-ph/0002126. Bibcode:2000RPPh...63..793B. 4885/63/5/2r3. A45 Metallic Hydrogen.

[44] Crane, L. (2017). "Metallic hydrogen finally made in lab at mind-boggling pressure". New Scientist. [Online] Available: https://www.newscientist.com/article/2119442-metallichydrogen-finally-made-in-lab-at-mind-boggling-pressure/.

[45] Lemmonick, S. (2017). "There's Reason To Be Skeptical About Metallic Hydrogen". Forbes, [Online] Available: https://www.forbes.com/sites/samlemonick/2017/01/27/theresreason-to-be-skeptical-about-metallichydrogen/\#666df570343f.

[46] Ashcroft, N. W. (2000). "The hydrogen liquids". Journal of Physics: Condensed Matter. 12 (8A): 129. Bibcode:2000JPCM...12..129A. doi:10.1088/0953$8984 / 12 / 8 \mathrm{~A} / 314$

[47] Zurek, E.; et al. (2009). "A little bit of lithium does a lot for hydrogen". Proceedings of the National Academy of Sciences. 106 (42): 17640-3. Bibcode:2009PNAS..10617640Z.

[48] D. Dolgov (1991) Baryogenesis in the Universe doi: 10.1007/978-94-011-3410-1 26. 
[49] Bonhoeffer KF, Harteck P (1929). "Para- and ortho hydrogen". Zeitschrift für Physikalische Chemie B. 4 (1-2): 113-141.

[50] (2003) Ortho and Para hydrogen (Spin Isomers Of hydrogen), chemistryworks.net [Online] Available: http://www.chemistryworks.net/2013/09/ortho-and-parahydrogen.html.

[51] (2012) Electrodynamics and Spacetime Geometry, BuffaloEdu [Online] Available: http://www.physics.buffalo.edu/phy514/w01/index.html.

[52] Pappas, S. (2013) Why do atoms get smaller across a period?, Reference.com [Online] Available:

https://www.reference.com/science/atoms-smaller-acrossperiod-88b34948679aa71f.

[53] Mysteriously Shrinking Proton Continues to Puzzle Physicists, LIVESCI=NCE [Online] Available: $\mathrm{http}: / / w w w . l i v e s c i e n c e . c o m / 28707$-shrinking-proton-puzzlenew-experiments.html.

[54] Mohit, $j$ (2015) The Mysterious World of Fundamental Particles: Cosmic Beginnings, ASIN: B00IILWVLO.

[55] The Delta Baryon, Hyperphysics, [Online] Available: http://hyperphysics.phy-astr.gsu.edu/hbase/Particles/delta.html.

[56] Willyard, C, Baryons - Delta Particles, Amsler et al. (Particle Data Group) (2008). "Review of Particle Physics". [Online] Available: http://www.whillyard.com/science-pages/delta.html.

[57] Adelberger, Eric G.; et al. (2011). "Solar fusion cross sections. II. The pp chain and CNO cycles". Reviews of Modern $\begin{array}{llll}\text { Physics. } & 83 & \text { (1): } 201 . & \text { arXiv:1004.2318. }\end{array}$ Bibcode:2011RvMP...83..195A. doi:10.1103/RevModPhys.83.195.

[58] N. E. Holden (2004). "Table of the Isotopes". In D. R. Lide. CRC Handbook of Chemistry and Physics (85th ed.). CRC Press. Section 11. ISBN 978-0-8493-0485-9.

[59] Tate, $K$ (2014) Proton Fusion, the Sun's Power Source, Explained (Infographic), space.com [Online] Available: http://www.space.com/26956-proton-fusion-sun-powersource-infographic.html.

[60] A2_1 Basic Ideas and Concepts in Nuclear Physics: An Introductory Approach, Third Edition K. Heyde Taylor \& Francis 2004. Print ISBN 978-0-7503-0980-6. eBook ISBN 978-1-4200-5494-1. DOI: 10.1201/9781420054941.ch2.

[61] B10Harp, D (2016) The Shadow Universe of Dark Matter Life, ISBN: 1523613947.

[62] A34 1Dorin N Poenaru et al. "Cold fission as heavy ion emission", Zeitschrift für Physik A, Springer Berlin / Heidelberg, Vol 328, No 3 / 1987, doi:10.1007/BF01290499, pp. 309-314.

[63] Ionization Energy, LibreTexts.org NCE [Online] Available: https://chem.libretexts.org/Core/Physical_and_Theoretical_Ch emistry/Physical_Properties_of_Matter/Atomic_and_Molecul ar_Properties/Ionization_Energy.

[64] Buzzle, The Concept of Negative Energy in Physics Simplified for You [Online] Available: http://www.buzzle.com/articles/the-concept-of-negativeenergy-in-physics-explained.html.

[65] NAAP Labs (2009).Hydrogen Atom "Energy Levels". University of Nebraska Lincoln [Online] Available: http://astro.unl.edu/naap/hydrogen/levels.html.
[66] Wolchover, $N$ (2011) What's the Total Energy In The Universe? [Online] Available: $\mathrm{http}: / /$ www.livescience.com/33129-total-energy-universezero.html.

[67] Negative Energy is Theorized as Dark Photons [Online] Available: https://en.wikipedia.org/wiki/Dark_photon.

[68] Carroll, Sean M. (October 29, 2008). "Dark photons". [Online] Available:

http://www.preposterousuniverse.com/blog/2008/10/29/darkphotons/.

[69] Bryman, D (2014) New Light Shed on Dark Photons [Online] Available: http://physics.aps.org/articles/v7/115.

[70] Koberlein, B (2016), Dark Matter, Dark Photons An the Fifth Force [Online] Available:

http://www.forbes.com/sites/briankoberlein/2016/08/29/darkmatter-dark-photons-and-the-fifth-force/.

[71] David C. Catling and Kevin J. Zahnle, (2009) The Planetary Air Leak, Scientific American [Online] Available: http://faculty.washington.edu/dcatling/Catling2009_SciAm.pd f.

[72] Andrew Nicholson (2013), Unifying Hinduism: Philosophy and Identity in Indian Intellectual History, Columbia University Press, ISBN 978-0231149877.

[73] Hydrogen as a Medical Gas (2016) Molecular Hydrogen Foundation [Online] Available:

http://www.molecularhydrogenfoundation.org/hydrogenemerging-medical-gas/.

[74] Molecular Hydrogen: An Emerging Medical Gas With Therapeutic Potential (2016) Molecular Hydrogen Foundation [Online] Available: http://www.molecularhydrogenfoundation.org/what-ismolecular-hydrogen/.

[75] Diana H. Barnes, Steven C. Wofsy, Brian P. Fehlau, and Elaine W. Gottlieb, James W. Elkins, Geoffrey S. Dutton, and Paul C. Novelli (2003) Hydrogen in the atmosphere: Observations above a forest canopy in a polluted environment. JOURNAL OF GEOPHYSICAL RESEARCH, VOL. 108, NO. D6, 4197, doi:10.1029/2001JD001199, 2003.

[76] How to Get the Benefits of Molecular Hydrogen (2013) Molecular Hydrogen Foundation [Online] Available: $\mathrm{http}: / / \mathrm{www} . \mathrm{molecularhydrogenfoundation.org/core-}$ information/how-to-get-the-benefits-of-molecular-hydrogen/.

[77] Negative Ions Create Positive Vibes WebMD [Online] Available: http://www.webmd.com/balance/features/negativeions-create-positive-vibes.

[78] Negative Ion Benefits for Your Health Negative Ionizers [Online] Available: http://negativeionizers.net/negative-ionsbenefits/.

[79] McDowell, Natasha (2003). "Air ionisers wipe out hospital infections". The New Scientist (Daily news). [Online] Available: https://www.newscientist.com/article/dn3228-airionisers-wipe-out-hospital-infections/.

[80] The International System of Units (SI), ed. Barry N. Taylor, NIST Special Publication 330, 2001. [Online] Available: https://www.nist.gov/sites/default/files/documents/pml/div 684/fcdc/sp330-2.pdf A33 Cold Fusion Hydrogen Production. 
[81] Hydrogen Compressibility at different temperatures and pressures, U.S. Department of Energy [Online] Available: http://hydrogen.pnl.gov/hydrogen-data/hydrogencompressibility-different-temperatures-and-pressures.

[82] Hydrogen Analysis Resource Center, U.S. Department of Energy [Online] Available: http://hydrogen.pnl.gov/hydrogendata/hydrogen-properties.

[83] McCarthy, J. (1995). "Hydrogen". Stanford University. [Online] Available: http://wwwformal.stanford.edu/jmc/progress/hydrogen.html.

[84] Ogden, J.M. (1999). "Prospects for building a hydrogen energy infrastructure". Annual Review of Energy and the Environment. 24: 227-279. doi:10.1146/annurev.energy.24.1.227.

[85] Weimer, $\mathrm{Al}$ (2005). "Development of solar-powered thermochemical production of hydrogen from water" (PDF). Solar Thermochemical Hydrogen Generation Project. [Online] Available: https://www.hydrogen.energy.gov/pdfs/review05/pd28_weime r.pdf.

[86] Perret, R. "Development of Solar-Powered Thermochemical Production of Hydrogen from Water, DOE Hydrogen
Program, 2007" (PDF). [Online] Available: https://www.hydrogen.energy.gov/pdfs/progress07/ii_f_1_perr et.pdf.

[87] Alternative Fuels Data Center: Hydrogen Basics, www.afdc.energy.gov [Online] Available: http://www.afdc.energy.gov/.

[88] Colella, W. G. (2005). "Switching to a U.S. hydrogen fuel cell vehicle fleet: The resultant change in emissions, energy use, and greenhouse gases.".

[89] Journal of Power Sources. 150 (1/2): 150-181. doi:10.1016/j.jpowsour.2005.05.092.

[90] Fuel Properties Comparison (PDF). energy.gov. Alternative Fuels Datacenter [Online] Available: http://www.afdc.energy.gov/fuels/fuel_comparison_chart.pdf.

[91] U.S. Energy Information Administration (EIA) - Annual Energy Review". Eia.doe.gov [Online] Available: https://web.archive.org/web/20100506022627/http:/www.eia.d oe.gov/emeu/aer/pecss_diagram.html. 